Letter to the Editor

\section{iMAX: A new tool to assess peripheral motor axonal hypoexcitability}

In routine electrodiagnostic procedures, classical parameters allow assessment of motor unit loss/reinnervation (CMAP amplitude) and motor conduction slowing (motor distal latency, motor conduction velocity, F waves), but none evaluates peripheral motor axon hypoexcitability. Yet everyone has experienced that in a demyelinating neuropathy it is often necessary to increase above normal the amount of current to obtain a supramaximal motor response. Excitability properties of human peripheral nerves can be assessed by various neurophysiological methods (Brismar, 1985; Kiernan et al., 2000; Burke et al., 2001), but they are not often used in daily practice because they are time consuming and they require a specific collection system and software.

The median nerve innervated thenar muscles were studied here with classical motor nerve conduction settings. The ground and recording electrodes consisted of pre-gelled disposable surface electrodes (Alpine Biomed, REF 9013L0453). The recording electrode was placed over the thenar eminence in close proximity to the muscle endplates halfway between the midpoint of the distal wrist crease and the first metacarpophalangeal joint. The reference electrode was placed over the proximal phalanx of the thumb. The ground electrode was placed over the ventral part of the forearm. All data were collected by the same investigator using a Keypoint G3 EMG machine (Natus Medical Incorporated). Compound motor responses were evoked by $1 \mathrm{~ms}$ constant current square waves through bipolar surface stimulation with two $7 \mathrm{~mm}$ diameter felt tip pads, $2.3 \mathrm{~cm}$ apart (Natus Medical Incorporated, REF 9013L0362). Despite the fact that previous studies showed that motor nerves have lowest threshold with a stimulus duration of typically $0.2 \mathrm{~ms}$, while sensory nerves can be excited at lowest threshold with a stimulus duration of $1.0 \mathrm{~ms}$ (Panizza et al., 1992), we used the longest stimulus duration of $1 \mathrm{~ms}$ in order to have if necessary the greater amount of current and to avoid technical limitation in the case of a severe motor axon hypoexcitability. The cathode was $2 \mathrm{~cm}$ proximal from the distal wrist crease and the anode was $2.3 \mathrm{~cm}$ proximal from the cathode. The bandpass filter setting was set from 2 to $5000 \mathrm{~Hz}$. In accordance with Ohm's law (voltage $=$ current $\times$ resistance), skin impedances under the cathode, anode and ground electrode were systematically measured and kept less than $20 \mathrm{k} \Omega$ by gently abrading the skin and wiping it with alcohol. The hand temperature was maintained above 30 ${ }^{\circ} \mathrm{C}$. The iMAX was the minimum stimulus intensity to elicit a maximal compound muscle action potential (CMAP) amplitude.

The motor threshold (minimum intensity required to evoke a motor response of at least $100 \mu \mathrm{V}$ of amplitude) was first measured. Then, the iMAX determination was a 3-step procedure. Firstly, the stimulus intensity was gradually and manually (about one stimulus every $2 \mathrm{~s}$ ) increased, in increments of $1 \mathrm{~mA}$, from the motor threshold to the intensity at which the CMAP amplitude, measured from baseline to the first negative peak, no longer increased (with a $0.1 \mathrm{mV}$ precision). One stimulus at the latter intensity, increased by $50 \%$ (supramaximal stimulus), was applied to be sure that the CMAP amplitude did not increase any further. Secondly, the stimulus intensity was decreased, in decrements of $0.1 \mathrm{~mA}$, until a CMAP amplitude reduction was obtained. Finally, the stimulus intensity was again increased by one or few increments of $0.1 \mathrm{~mA}$ and when the maximum CMAP amplitude (determined at the first step) was reached, the corresponding stimulus intensity was defined as iMAX.

This up-down-up stimulus intensity procedure was applied to 30 healthy controls (mean age: $44 \pm 19$ years), 9 patients with a chronic axonal neuropathy involving the median nerve motor territory (age ranged from 23 to 70 years), 5 patients with a CharcotMarie-Tooth 1A (CMT1A) (age ranged from 9 to 42 years) and 6 patients with an acute polyradiculoneuritis (age ranged from 23 to 80 years), 4 patients with classical Guillain-Barré syndrome (GBS) and 2 patients with an acute motor axonal neuropathy (AMAN).

In controls, the mean motor threshold was $1.6 \pm 0.7 \mathrm{~mA}$ and the mean iMAX was $4.6 \pm 1.2 \mathrm{~mA}$. The mean skin impedance was $7 \pm 3$ $\mathrm{k} \Omega$ under anode, $10 \pm 3 \mathrm{k} \Omega$ under cathode and $5 \pm 3 \mathrm{k} \Omega$ under the ground electrode. Neither motor threshold data, nor skin impedance data correlated with iMAX data (tested by the Spearman correlation coefficient). Upper limits of normal (ULN) (mean +2 SD) were $3.0 \mathrm{~mA}$ for the motor threshold and $7.0 \mathrm{~mA}$ for iMAX (Fig. 1). The motor threshold was systematically increased in CMT1A patients (between 4.3 and $15.0 \mathrm{~mA}$ ). In other patients, the motor threshold was less than $3.0 \mathrm{~mA}$, except in 2 patients with classical GBS (4.6 and $7.9 \mathrm{~mA}$ ). In patients with axonal chronic neuropathies, iMAX remained below ULN. In patients with CMT1A, iMAX was clearly increased (between 16.2 and $59.8 \mathrm{~mA}$ ). In patients with classical GBS, iMAX was also clearly and early increased (between 7.7 and $65.3 \mathrm{~mA}$ ). In AMAN, iMAX was below the ULN in one patient (after 15 months since disease onset), and increased at day $10(11.9 \mathrm{~mA})$ and below the ULN at day 24 (3.7 $\mathrm{mA}$ ) in the other patient (Fig. 1).

We propose iMAX as a promising and sensitive parameter to assess peripheral motor axonal hypoexcitability in routine practice, with classical recording and stimulating electrode settings. To our knowledge, this method has not yet been described in the literature. This parameter may be derived in a few minutes at each stimulation point for every peripheral motor nerve. Interestingly, iMAX is clearly distinct from a usual threshold measure as it measures the motor axons with the highest threshold of all axons. From a clinical point of view, iMAX might be helpful to distinguish axonal versus demyelinating polyneuropathies (Fig. 1) and possibly acquired demyelinating (iMAX increased at some time points, 


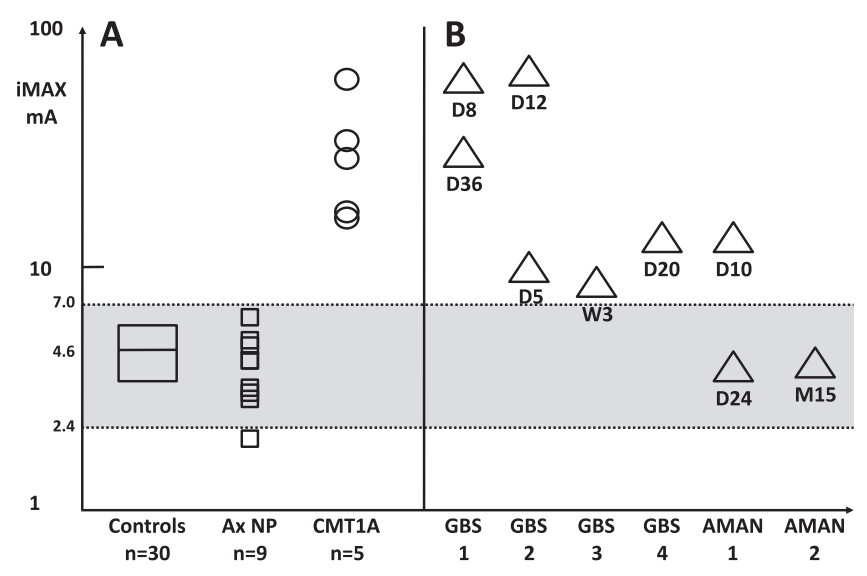

Fig. 1. iMAX results. (A) In 30 controls (box: mean \pm SD) and 14 patients with chronic axonal neuropathy involving the median nerve motor territory (Ax NP) (black squares, $n=9$ ) or Charcot-Marie-Tooth 1A (CMT1A) (black circles, $n=5$ ). B) In 6 patients (black triangles) with a classical Guillain-Barré syndrome (GBS) $(n=4)$ or an acute motor axonal neuropathy (AMAN) $(n=2)$. Three patients were evaluated only once (GBS 3, GBS 4 and AMAN 2) and twice for the 3 others (GBS 1 , GBS 2 and AMAN 1). $\mathrm{D}=$ day, $\mathrm{W}=, \mathrm{M}=$ month (since the disease onset). Dotted lines $=$ upper $(7.0 \mathrm{~mA})$ and lower $(2.4 \mathrm{~mA})$ normal limits of iMAX (mean $\pm 2 \mathrm{SD})$. Y axis = logarithmic scale.

but remaining within normal limits at others) versus inherited demyelinating polyneuropathy (iMAX increased in all cases).

\section{Conflict of interest}

Christophe Milants, Karim Benmouna and François C Wang have no conflicts of interest.

\section{References}

Brismar T. Changes in electrical threshold in human peripheral neuropathy. J Neurol Sci 1985;68:215-23.

Burke D, Kiernan MC, Bostock H. Excitability of human axons. Clin Neurophysiol $2001 ; 112: 1575-85$

Kiernan MC, Burke D, Andersen KV, Bostock H. Multiple measures of axonal excitability: a new approach in clinical testing. Muscle Nerve 2000;23:399-409. Panizza M, Nilsson J, Roth BJ, Basser PJ, Hallett M. Relevance of stimulus duration for activation of motor and sensory fibers: implications for the study of H-reflexes and magnetic stimulation. Electroencephalogr Clin Neurophysiol $1992 ; 85: 22-9$

Christophe Milants

Karim Benmouna

François Charles Wang *

Department of Neurophysiology, CHU Sart Tilman B35, 4000 Liège,

Belgium

* Corresponding author at: CHU Sart Tilman B35, 4000 Liège,

Belgium.

E-mail addresses: cmilants@chu.ulg.ac.be (C. Milants),k.benmouna@ chu.ulg.ac.be (K. Benmouna), fc.wang@chu.ulg.ac.be (F.C. Wang)

Available online 7 October 2017 